\title{
LA PROBLEMÁTICA DEL CAMBIO SEMÁNTICO EN LA LENGUA COMO SISTEMA DE VALORES: APROXIMACIÓN EPISTEMOLÓGICA
}

\author{
JUAN LUIS JimÉnEZ RUIZ \\ (Universidad de Alicante)
}

\begin{abstract}
RESUMEN
The present paper constitutes an attempt to establish the parameters in order to place a general view of change meaning within the context of Subject Linguistic, and to organize in adequate epistemological channnels the result achieved.
\end{abstract}

Sin lugar a dudas, el léxico de una lengua está sometido a una serie de presiones que modifican su significado a lo largo de la historia. Los ejemplos serían muy numcrosos y no es el momento ahora de traerlos a colación: sirva, simplemente, como mera prueba ilustrativa el caso de retrete, cuyo significado no sería entendido de la misma manera por un hombre del siglo xvi que por uno del siglo $\mathrm{xx}$.

Ello justifica que la reflexión sobre el léxico de una lengua deba considerar también, junto a los aspectos sistemáticos y sincrónicos, las consideraciones diacrónicas sobre la misma. En este sentido, vamos a repasar en este trabajo los distintos tipos de análisis lexical, precisando los diferentes modelos sobre el léxico y su evolución, como primer jalón descriptivo de las formulaciones hipotéticas sobre el cambio semántico en el marco epistemológico de la Lingüística del Sujeto.

1 Efectivamente, el significado de retrete, de incluir el semema 'cuarto pequeño en la casa o habitación destinado a retirarse' que da el Diccionario de Autoridades, recoge hoy en el D.R.A.E. (1992): 'aposento dotado de las instalaciones necesarias para orinar y evacuar el vientre', con lo que queda confirmado el hecho de que el léxico no permanece inalterable a lo largo del tiempo. 


\section{El análisis del léxico}

\subsection{Dos tipos de análisis}

La disciplina de los significados se extravió desde Aristóteles porque se creyó que los significados eran una función de las formas lingüísticas y que las formas linguísticas tenían un significado. Tal es la opinión de T. de Mauro², quien defiende la tesis de que los significados deben considerarse como un resultado y como una función del acto significador y no al revés; es decir, que no son las palabras sino los hombres quienes significan por medio de ellas, y que la garantía del acto significador no reside en las propias formas lingüísticas, sino en las sociedades que las utilizan ${ }^{3}$. Y como las sociedades están en el continuo cambio que la percepción diacrónica permite observar, el análisis sincrónico del léxico se ha visto completado con la visión diacrónica.

Con todo, no debe desprenderse de esta afirmación la clave sociológica como mera explicación de la evolución lingüística, aunque sí la importancia de la noción de sociedad ${ }^{4}$ como principio organizativo y configurador de los hechos linguísticos. No en balde, el proceso epistémico que parecía establecer una relación de presuposición mutua e interdependencia entre las perspectivas sincrónica y diacrónica de acercamiento objetual, posibilitó en algunos casos desarrollos paralelos e independientes. Recuérdese, por ejemplo, que durante el siglo xix la descripción fue reemplazada por la historia, y ésta última reinó en la lingüística hasta comienzos del siglo $\mathrm{xx}$, en el que Saussure distinguió lo sincrónico de lo diacrónico.

Por ello, quizá, se produjo la separación metodológica, alentada además por los resultados a veces contradictorios e irreconciliables (el más conocido es la etimología popular) de ambas propuestas.

Sin embargo, el análisis del léxico requiere la combinación de ambos planteamientos ya que ciertas condiciones sincrónicas sólo pueden ser reconocidas una vez que han ocurrido, a la luz de las consecuencias diacrónicas que emanan de ella; además, el trasfondo de ciertos cambios semánticos únicamente puede detectarse mediante el estudio cuidadoso del estado de la lengua antes del cambio. Por todo ello, se hace necesario un acercamiento tanto sincrónico como diacrónico que nos permita precisar una serie de cuestiones que, de otra manera, habrían pasado inadvertidas 5 .

2 Cf. T. de Mauro, Sentantica, pág. 10.

3 Así, el autor propone una teoría de la significación que recupera el sociologismo semántico de Saussure, la teoría de Bloomfield de la significación explicitada solamente por la situación, los aforismos de Wittgenstein, y las ideas de Humboldt y Whorf.

4 Cf. L. Rodríguez Zúñiga, «Desarrollo», apud S. del Campo, Sociología, pp. 15-56. $7-35$.

Cf. S. Ullmann, Lenguaje, pp. 60-66, y el prólogo de A. Alonso al Curso de Saussure, pp. 
El problema estriba en la concepción epistemológica en la que se va a basar el desarrollo metodológico de tales cuestiones ${ }^{6}$, puesto que, aunque la semántica moderna haya abandonado el espejismo de las leyes universalmente válidas y se haya constituido en lo que Ullmann ${ }^{7}$ denomina semántica pansincrónica (que estudiaría los rasgos generales de la estructura semántica, por ejemplo, la relación entre la polisemia y la frecuencia de las palabras), y semántica pandiacrónica (que estudiaría las formas del cambio semántico ampliamente predominantes, tales como las metáforas antropomórficas o las transferencias de lo concreto a lo abstracto), las propuestas teoricometodológicas de la lingüística objetual basadas en el paradigma realista de descripción formalista no agotan la totalidad del objeto/sujeto estudiado — la vertiente trascendental-, sólo aprehensible a través de propuestas sujetuales más cercanas al paradigma idealista de la interpretación ${ }^{8}$.

$Y$ es que, de hecho, tal y como reconociera hace ya años F. Restrepo ${ }^{9}$ la palabra no es una entidad estable, es la unidad linguística por excelencia que recoge de manera certera la visión de cada sociedad y las presiones que recibe la misma, en un proceso de tensión dialéctica que la lleva a evolucionar variando su significado a lo largo del transcurso del tiempo ${ }^{10}$.

Ello nos mueve a considerar las palabras, siguiendo las propuestas de L. Zgusta, como unidades interpersonales del lenguaje (signos de un sistema lingüístico), que el hablante utiliza para hacer oraciones en las que las palabras hacen referencia a partes del mundo extralingüístico ${ }^{11}$. Sin embargo, el hecho de que el significado de estas palabras puede modificarse, ampliándose o restringiéndose a lo largo de la historia ${ }^{12}$, nos hace ver la utopía que supondría una visión total de la estructura del léxico de una lengua, por su propio dinamismo y amplitud.

G Evidentemente, porque el punto de vista que se adopte para la determinación de nuestro objeto de estudio va a posibilitar los diferentes planos de acercamientos reflexivos al mismo; en el caso que nos ocupa, desde el punto de vista diacrónico, y en el caso de nuestro trabajo «Caracterización semántica», pp. 441-454, desde el punto de vista sincrónico.

Cf. S. Ullmann, Lenguaje, pág. 73.

\& Su desarrollo tanto teórico como metodológico puede verse en nuestro trabajo Semántica.

- Cf. F. Restrepo, Senántica, pág. 29.

10 Mientras el «vocabulario» de otros tipos de códigos semiológicos es estable, en el caso de las lenguas nos encontramos con un conjunto altamente inestable, sobre todo por la creatividad, que se manifiesta como una continua oscilación potencial y efectiva; cf. T. de Mauro, Minisemántica, pág. 113.

11 A pesar de ello, aunque las palabras tengan un significado estabilizado, no poseen una experiencia abstracta, sino que son usadas; de ahí la diferencia entre la significación actual, la significación en el pasado y el significado de una unidad léxica fuera de cualquier contexto; $\mathrm{cf}$. L. Zgusta, «Lexical Meaning», apud Manual, pp. 21-118.

12 Véase como ejemplo ilustrativo el trabajo de M. A. García Asensio e I. Yufera, «Evolución semántica», pp. 359-373. 
Ante este problema, la lingüística objetual y sujetual han reaccionado de diferente manera. En el primer caso, los lingüistas del objeto, presos del cientificismo y del criterio de verdad como único prisma organizador de la realidad lingüística, comenzaron rechazando los estudios semánticos de su ámbito disciplinario.

Posteriormente, otro grupo de lingüistas, aunque conscientes de que todavía faltan conocimientos exactos sobre las estructuras léxicas y semánticas, sostienen que un hecho indudable es que el semema representa un conjunto de semas estructurados de un modo específico. No es casual, por tanto, que el estudio de las estructuras semánticas se haya dedicado primero a las estructuras del léxico y, sólo en tiempos recientes, a las estructuras inmanentes, a los sememas, ya que en algunos sectores del léxico, las estructuras parecían más evidentes y para la descripción exacta de las estructuras del semema hacía falta un análisis de constituyentes semánticos con materiales empíricos extensos ${ }^{13}$.

Ello posibilitó el análisis somero de todos los rasgos del léxico de una lengua, con la ayuda de los ordenadores. Hoy en día, prestigiosas universidades del mundo dedican gran parte de sus esfuerzos a estas investigaciones. Podemos citar, entre otras, el laboratorio de análisis lexicográfico de la Universidad de Besançon (en el que destaca la figura de B. Quémada); el Centro de búsqueda para el Tesoro de la Lengua francesa de Nancy (con P. Imbs a la cabeza); el Centro de Lingüística automática del Instituto italiano en la Universidad de Utrecht (donde se realizan los grandes trabajos estructurales sobre el léxico dirigidos por M. Alinei); el Centro de Lexicografía italiana de la Academia della Crusca; el Centro Nacional Universitario de Cálculo electrónico de la Universidad de Pisa (con los importantes trabajos de Zampolli); el Instituto de Lexicología francesa de la Universidad de Lieja; etc.

En España destacan los trabajos llevados a cabo por el Departamento de Filología Española I de la Universidad de Málaga dirigidos en aquel entonces por M. Alvar Ezquerra ${ }^{14}$.

Sin embargo, junto a la valoración histórica del sistema lingüístico (ya sea desde el punto de vista estrictamente sincrónico o como suma de sincronías sucesivas) se hace necesaria también la aprehensión del sistema de valores lingüísticos ${ }^{15}$, valores que se dan en diferentes dominios de la aplicación empírica glotológica.

En este sentido, la lingüística del sujeto aporta el mecanismo exegético complementario para llevarnos a una comprensión integral que nos permita

13. Cf. G. Wotjak, Investigaciones, pp. 85-88.

14 Al respecto es interesante M. Alvar Ezquerra et alii. Industrias, Corpus. También puede verse su trayectoria profesional en nuestro trabajo «Proyecto lexicográfico», pp. 493-502.

15 Cf. M. Crespillo, «Miseria», pág. 282. 
aprehender de manera globalizante el proceso de evolución lingüística, para poder así interpretar el presente a partir del pasado y explicar el pasado desde la reflexión del presente ${ }^{16}$.

De ahí que, metodológicamente, junto a la distinción ya clásica entre denotación (relación existente entre las palabras y las partes del mundo extralingüístico a las cuales designa) y connotación (cúmulo de componentes de significado que añaden un valor contrastado a la función de designación básica) debamos añadir la propuesta de Zgusta ${ }^{17}$ de esfera de aplicación de la palabra, que es distinta de la connotación, puesto que se trata de las posibilidades individuales de uso de la palabra en un dominio o en otro.

Por tanto, el estudio diacrónico de las esferas de aplicación que actualizan los distintos sememas a través de una serie de semantemas, será el que permita la aproximación a la evolución diacrónica del léxico estudiado. Dicho de otra forma, el estudio histórico de los semantemas que dan forma léxica a las esferas de aplicación, en el marco de los distintos sistemas en los que puede estructurarse el léxico a través de la historia, constituye el objeto que la reflexión diacrónica debe sistematizar. Veamos, seguidamente, algunas propuestas de caracterización objetual.

\subsection{Modelos sobre el léxico}

La lexicología en cuanto disciplina encargada del estudio de la forma del plano del contenido absoluto, designativo o predicativo del signo lingüístico, liene por objeto final la comprensión del hecho léxico, tanto en el nivel de las estructuras (léxicos, vocabularios) ${ }^{18}$ como de las unidades (palabras, etc.).

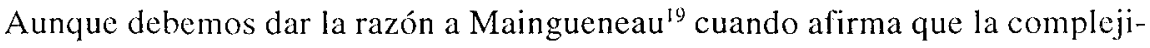
dad, el entrecruzamiento y la fluidez de las estructuras léxicas han desalentado a muchos investigadores ante la tarea de realizar un análisis lexicológico de la lengua ${ }^{20}$, también es cierto que existen dominios en los que sí es posible la estructuración.

Siguiendo las distinciones propuestas por R. Trujillo ${ }^{21}$, la lexicología debe tratar, por tanto, de la forma en sus relaciones con la substancia del contenido o,

16 Véase para ello, W. Labov, «Presente», apud Il continuo, pp. 121-158.

17 Cf. L. Zgusta, Manual, pp. 27 y ss.

18 Entiéndase léxico como los términos de una lengua, y vocabulario como los términos de un discurso. El vocabulario, efectivamente, está ligado a un texto y supone la existencia de un léxico del que sólo es una muestra; cf. Ch. Müller, Estadística, pp. 230-231.

19) Cf. D. Maingueneau, Análisis, pág. 55.

20 Hasta el punto de que, como reconoce Martinet, muchos lingüistas han negado el carácter sistemático de la lengua al léxico; cf. A. Martinet, «EI léxico», apud Lingüística, pp. 229-243.

21 Cf. R. Trujillo, «Gramática», pp. 103-109. 
en palabras de J. Mondéjar ${ }^{22}$, del léxico desde un doble punto de vista: desde el que lo concibe como una estructura, y desde el que lo hace como un conjunto de doble naturaleza; a saber, material (expresión) y significativa (contenido).

En definitiva, se trata de la consideración de la lexicología como el estudio del origen, forma y significado de las palabras desde el punto de vista general y científico ${ }^{23}$.

A pesar de la confusión que la lexicología ha tenido con otras disciplinas ${ }^{24}$, lo que ha indeterminado tanto su objeto como - lo que es más importante-el método que debe seguir en su análisis, Matoré25 la precisa de manera clara como la disciplina de carácter sintético que estudia los hechos de civilización. En este sentido, el vocabulario no sólo es reflejo o producción mecánica de la realidad, sino un determinante de la misma. Sus límites estarían en la incapacidad para expresar de forma adecuada la esencia profunda de las cosas, y el aspecto más individual del $\mathrm{yo}^{26}$.

A. Rey, por su parte, distingue tres maneras de considerar el léxico según las necesidades metodológicas:

- como el conjunto de los morfemas de una lengua (punto de vista adoptado por gran parte de los funcionalistas y distribucionalistas);

- como el conjunto de las palabras (morfemas libres que integran el sintagma);

$-\mathrm{y}$, finalmente, como un conjunto indeterminado, pero finito, de elementos, en oposición a los que realizan directamente funciones gramaticales.

Partiendo de la consideración del léxico en la tercera acepción señalada, lo concebimos como la irregularidad fundamental, frente a las regularidades de la morfosintaxis y de la fonología.

El factor de la vacilación y de las aplicaciones divergentes es de gran importancia pues, a veces, es muy difícil decidir si una significación es sistemática u ocasional. En este sentido, Zgusta ${ }^{27}$ mantiene que es tarea del lexicógrafo indicar la historia del significado y del cambio, mientras que M. Alvar Ezquerra ${ }^{28}$

22 Nos referimos al prólogo de la obra de M. del M. Espejo Muriel, Colores, en el que J. Mondéjar, ante las diferentes concepciones que de algunas disciplinas se ha tenido a lo largo de la historia, intenta fijar lo que debe entenderse por semántica, lexicología, onomasiología y semasiología; cf. J. Mondéjar, «Palabras», apud M. del M. Espejo Muriel, Colores, pág. 11.

23. Es la propuesta que puede verse en el capítulo II «Lexicología y lexicografía» de la obra de J. Casares, Lexicografía.

24 Piénsese, por ejemplo, en disciplinas afines tales como la Estilística, Morfología, Gramática, Psicología y Semántica, por poner unos casos.

25 Cr. G. Matoré, Méthode, pp. 47 y ss.

26 En el fondo, como reconoce M. Alvar Ezquerra, se trataría de una sociología a través de las palabras; cf. M. Alvar Ezquerra, Lexicologia, pp. 39-44.

${ }_{27} \mathrm{Al}$ respecto puede verse L. Zgusta, Manual, pp. 47-60.

$2 \times$ Cf. M. Alvar Ezquerra, Lexicología, pág. 85. 
piensa que este estudio no corresponde al lexicógrafo, sino al lexicólogo. Sea uno o sea otro, lo que es bien cierto es que la historia del significado permitirá advertir no sólo la posible evolución de $\operatorname{los} \operatorname{mismos}^{29}$, sino también la del sistema de valores.

Consecuentemente, no se trata de situar el significado en la historia sino de realizar la historia del significado ${ }^{30}$ mediante un ejercicio de reproducción del lenguaje sujeto en la lengua objeto que permita no sólo la visión semántica de un orden sino también la aprehensión de ese mismo orden a través del cambio semántico ${ }^{31}$.

\section{EI problema del cambio semántico}

Desde los padres fundadores de la semántica hasta S. Ullmann y P. Guiraud, se han incluido bajo el rótulo general de cambio semántico gran cantidad de fenómenos diferentes. Es evidente que las palabras varían de significado, ampliando o restringiendo su zona de significación, como es perceptible a lo largo de la historia de la lengua. Esta evolución, por lo general, va unida a una diferencia de grado tanto en la percepción de la realidad extralinguística como en la propia realidad. Sin embargo, no debe pensarse que la estructura de lo real es la que condiciona la estructura del contenido lingüístico, sino que el contenido linguístico es la estructuración que se da en la lengua a los datos de la experiencia de lo real. De ahí que, tal y como hemos manifestado, la propuesta teórica deba consistir en la aprehensión de la estructura de lo real (orden) a partir de la organización lingüística (semántica) de ese mismo orden y su proceso evolutivo plasmado en los cambios semánticos.

Según Trujillo ${ }^{32}$, estos cambios de sentido de las palabras, considerados en sí mismos, son los cambios semasiológicos, mientras que los que se consideran en cuanto fenómenos que guardan relación con un conjunto estructurado y que tienen su explicación en la evolución de esta estructura son los cambios semánticos ${ }^{33}$.

29 Un ejemplo muy interesante del cambio semántico concretamente en el léxico político puede verse en J. C. Garrido Medina, «Cambio semántico», apud M. Alvar (coor.), Lenguaje, pp. 136-146.

30 Véase al respecto nuestras reflexiones en «Significado», pp. 9 y ss.

${ }^{31}$ Una visión general del orden decimonónico y de las repercusiones epistémicas de su concepción semántica puede verse en nuestro trabajo «Carácter semántico», pp. 89-97.

32 Cf. R. Trujillo, Campo, pág. 37.

3. Nos referimos a los cambios en la lengua como reflejo de cambios en la realidad, frente a los cambios internos de la lengua. En el primer caso, dice Mondéjar que hay que hablar de cambios terminológicos (correspondientes a términos que designan hechos, objetos extralingüísticos), y en el segundo, de palabras (cuya definición es siempre interna y comparativa).

En el primer caso se trata del dominio de la onomasiología y en el segundo de la semasiología (propiamente lingüística). Existe pues un acuerdo conceptual con Trujillo, aunque no 
Y puesto que en nuestro ámbito disciplinario existe una falta de univocidad terminológica ${ }^{34}$ que, la mayoría de las veces, conduce a confusiones y a errores de comprensión, vamos a precisar estos términos, refiriéndonos con E. Coseriu ${ }^{35}$, a cambios semánticos cuando persiste el lexema y varía diacrónicamente el semema, y hablando de cambios léxicos cuando se mantiene el mismo semema pero cambia el lexema que lo manifiesta ${ }^{36}$.

El cambio semántico mantiene, pues, el lexema, pero el semema sustituye o suprime alguno de sus semas, convirtiéndose así en otro semema, aunque conserve alguno de sus semas anteriores ${ }^{37}$.

Una somera visión a lo largo de la historia sobre esta cuestión nos permite apreciar que la problemática del cambio semántico interesa a muchas escuelas. La mayoría de ellas, partiendo del juicio apriorístico de la regularidad básica del hecho lingüístico, consideran la lengua como un sistema de reglas en el que se producen una serie de cambios (excepciones a la regla). Mención aparte debe hacerse de la sociolingüística, que considera este fenómeno fundamental, hasta el punto que pretende construir un modelo a partir de la variación ${ }^{38}$.

En líneas generales, la observación de los cambios lingüísticos ha sido tarea de la lingüística histórica, que se ha ocupado de ellos en el terreno fónico, tratando, principalmente, de la comparación de las unidades para obtener reglas que constituyesen un sistema. Sin embargo, existe un rasgo -entre otros-que separa los cambios semánticos de los fonéticos: mientras que éstos se producen con cierta regularidad y dentro de ciertas tendencias particulares en cada lengua, los cambios semánticos no ofrecen ni la regularidad ni los rasgos específicos aludidos en la misma proporción. Tienen un carácter más universal, ya que afectan, puede decirse, a la parte más espiritual del lenguaje y más alejada de las peculiaridades de los diferentes grupos lingüísticos ${ }^{39}$.

Con todo, los cambios semánticos son, naturalmente, un tipo de cambio lingüístico y, en este sentido, ofrecen características comunes con los demás. La

terminológico; cf. J. Mondéjar, «Palabras», apud M. del M. Espejo Muriel, Colores, pp. 11-15. Una precisión de las nociones de semántica, onomasiología y semasiología puede verse en el excelente trabajo de M. Casas, "Caracterización», especialmente en las pp. 49-53.

${ }_{34}$ Cf. E. Coseriu, Lecciones, pp. 124 y ss.; J. Fernández Sevilla, «Sinonimia», pp. 41-55; o G. Salvador, Semántica, pp. 126-132; por poner unos casos.

35 Cf. E. Coseriu, «Hacia una semántica diacrónica estructural», apud Principios, pp. 11-87.

36 Sea cual fuere, debemos sostener con $\mathrm{R}$. Trujillo que debe hablarse de cambio de significado siempre que afecten a la estructura del contenido y no se reduzcan a nuevas substituciones de significantes o de significados; cf. R. Trujillo, Campo, pág. 37.

$37 \mathrm{Al}$ respecto es interesante J. Lyons, Semántica, pp. 253-315.

${ }_{38}$ Pueden citarse al respecto los trabajos en lingüística variacionista (secular linguistics) de W. Labov o los de Bailey, Bickerton, Cedergren, Sankoff, Trudgill, y Weinreich, entre otros; cf. F. Gimeno, Dialectología, pp. 148 y ss.

3. Una visión general al respecto puede verse en J. M. Anderson, Cambio. 
transmisión de las lenguas de una generación a otra no es nunca completamente fiel y, por lo tanto, se originan modificaciones o cambios en general.

Recordemos, por ello, aunque sea de manera somera, los distintos modelos teóricos sobre el cambio.

\subsection{Modelos teóricos sobre el cambio}

No debe pensarse que vamos a proceder, a continuación, a la descripción exhaustiva de las distintas formulaciones teóricas que, a lo largo de la historia de la lingüística, han descrito el fenómeno del cambio lingüístico ${ }^{40}$. Entre otras razones, porque la detallada, y hasta cierto punto, interesante descripción que podríamos realizar, sería en exceso larga, y la aportación específica poco relevante para la reflexión posterior. Sirva, por tanto, como mero recordatorio, que nos permita centrar el estado de la cuestión y la adopción crítica del sistema epistemológico que se pueda tomar.

Desde el clásico modelo histórico-genético que, a partir de la comparación entre las distintas lenguas, intentaba construir el parentesco entre las mismas, hasta el modelo transformatorio que explica la variación como un cambio de reglas, han sido muchos y muy variados los intentos de construir un aparato teórico que describiese el fenómeno del cambio lingüístico.

Uno de ellos fue el que culminó en la teoría de las ondas (Wellentheorie) de Schimidt (1872), para quien la relación entre las lenguas es el resultado de una disimilación semejante a la de las ondas en los dialectos del protoindoeuropeo. Por tanto, las lenguas no existirían independientemente sino en diasistemas que contactan por ondas. Sin embargo, no llega a explicarse ni el origen ni la regularidad del cambio.

Basado en el árbol genealógico (Stammbaumtheorie) de Schleicher, el modelo biológico explica la evolución de la lengua como un suceso natural dentro del marco de las especies. Está basado en el paralelismo entre algunas características de la biología y la lingüística; a saber, la tendencia a la regularidad y a la armonía, la irreversibilidad, y el hecho de que tanto los genes como los elementos lingüísticos se modifican por unos principios ${ }^{4 !}$. Sin embargo, en la biología los cambios tienden a perfeccionar el sistema, mientras en la lingüística no se sabe hasta que punto es esto cierto.

40 Una visión amplia y exhaustiva puede consultarse en W. Porzig, «Cambios lingüísticos», apud Lenguaje, pp. 294-357. También es interesante el recorrido histórico realizado por F. Gimeno, Sociolingiuistica, pp. 11 y ss.

${ }_{41}$ De hecho, como reconoce F. Gimeno, Dialectología, pp. 57 y ss., en la segunda mitad del siglo XIX los estudios lingüísticos se centraron en los aspectos físicos y biológicos del lenguaje, considerando, por ejemplo, los sonidos como un producto fisiológico semejante a otros fenómenos físicos, y sometido a leyes inmutables. 
Finalmente, el modelo dinámico de Martinet ${ }^{42}$ se aplica al sistema fonológico, cuya inestabilidad lleva a homogeneizar el sistema, lo que produce cambio. Se puede objetar a Martinet el rechazo del papel sintagmático de la significación, y la no consideración de los cambios morfológicos o sintácticos.

\subsection{Tipos de cambio}

La lengua se procura nuevos contenidos creando palabras nuevas y haciendo que palabras ya creadas adquieran sentidos diferentes al que ya tenían. En general, los cambios pueden ser de distintos tipos:

- cambios léxicos, que pueden darse: $1^{\circ}$, por adición, incorporación léxica que se produce debido a la necesidad de dar un nuevo nombre a lo que antes no lo tenía ${ }^{43}$, y por factores de moda, unidos al prestigio social y político de una lengua ${ }^{44} ;$ y $2^{\circ}$, por pérdida léxica, debido a que el objeto designado pierde su uso, lo que hace que deje de usarse también la unidad léxica, o que adopte un significado distinto ${ }^{45}$;

- cambios fonológicos, morfológicos y sintácticos, originados por adición, supresión, o cambio de regla.

En el cambio de significación el hablante juega un papel subordinado que se realiza por el hecho de que el oyente no tiene necesidad de tomar cada palabra en el sentido que tiene.

B. Sne $1{ }^{46}$, atendiendo al cómo y al porqué surgen los cambios semánticos, los clasifica en dos grandes grupos ${ }^{47}$ :

- cambio voluntario de la significación, producido cuando se le da a una palabra un sentido nuevo del que tenía o se crea una palabra nueva apoyándose en el empleo que mejor suene; éste cambio de significación es importante para

42 En general, el modelo de Martinet sobre el cambio se basa en un estado de perturbación constante del sistema, lo que origina su variación.

4.3 Véase al respecto el trabajo de T. M. Rossi, «Denominar», pp. 323-331.

44 Lo que entraña, obviamente, usos impropios y eufemísticos; cf. E. del Teso Martín, «Cambio semántico», pp. 183-204.

45. De hecho, la unión de las palabras con los objetos o nociones que designan obliga a que el cambio en las técnicas, instrumentos o ideas de una determinada época vaya unido a un cambio en el léxico de una lengua, ya sea mediante la desaparición de una palabra, la aparición de otras o el cambio en la designación de algunas. Por ello, como reconoce M. Alvar Ezquerra, Fornación, pág. 10 , junto al léxico patrimonial de una lengua, hay palabras que no existen desde siempre, ya sea porque se han formado a partir de otras o porque se han incorporado de manera novedosa. El léxico de la lengua española consta así de palabras heredadas (un 23\% del total), préstamos (un $41 \%$ ) y voces creadas (un 35\%). Sin embargo, la frecuencia de uso es muy distinta, ya que representan un $81 \%, 10 \%$ y $8 \%$ respectivamente, lo que demuestra el amplio uso que tiene el léxico heredado, aunque sea minoritario.

46 Cf. B. Snell, «Cambios de significación», apud Estructura, pp. 164-168.

${ }^{47} \mathrm{Al}$ respecto puede verse también la aportación de D. Geeraerts, «Semantic change», pp. 217-240. 
la evolución y formación de términos filosóficos;

- cambio involuntario de significación, producido cuando se le da una nueva definición a términos antiguos, con lo que pasan a emplearse en un nuevo sentido.

En general, podemos decir que existen distintas tendencias en la evolución significativa de las palabras, como puede ser el refuerzo del sentido abstracto, o el cambio en la idea de vinculación local a otra temporal y causal, por poner unos ejemplos, pero difícilmente se llega a un verdadero cambio lingüístico de significación total.

\subsection{Causas de los cambios semánticos}

Como sostiene F. Restrepo ${ }^{48}$, las palabras son representaciones de las cosas y expresión de las ideas y sentimientos. Si estos tres factores fueran invariables no habría razón para que las palabras cambiaran, pero como son variables y están sujetas a modificaciones, siguen el movimiento de estos tres elementos buscando expresarlos con mayor exactitud. Las causas de este «movimiento semántico», en palabras de Restrepo, son tres: las variaciones de las cosas, la modificación de los conceptos, y la desviación de la significación en las palabras.

Las variaciones en las cosas se deben a que algunas desaparecen, ya sea porque están relacionadas con usos y costumbres de antepasados o corresponden a civilizaciones muy antiguas; otras cosas nuevas van surgiendo por el aumento del caudal de nuestro conocimiento, aparición de instituciones, doctrinas, ciencias, etc.; en otros casos, se dan a las cosas ya conocidas nuevas formas y aplicaciones, lo que requiere una nueva palabra para su designación.

Estas variaciones de las cosas permiten el aumento de los conceptos y, consecuentemente, la modificación de los mismos. Entre las razones de esta variación, Restrepo comenta las siguientes: la aclaración de una idea confusa, y la mayor percepción de la realidad extralingüística ${ }^{49}$.

Finalmente, los movimientos de las palabras que acusan cambios semánticos se deben también a una desviación en la significación de las palabras, motivadas, en general, por causas lingüísticas, históricas, psicológicas, y sociales. Veamos cada una de ellas.

48 Cf. F. Restrepo, Semántica, pág. 29.

49 Efectivamente, lo que Restrepo llama distinción, análisis y coloración, permiten una mayor percepción de la realidad; en el primero de los casos, se trata de distinguir con una observación más atenta cosas más o menos semejantes que significábamos con un nombre general; con el análisis nos fijamos en las cosas que nos rodean y distinguimos sus diversas cualidades y partes; finalmente, las ideas sobre los objetos poseen tintes variados (coloración), por lo que es necesario buscar una nueva expresión para manifestarlos; cf. F. Restrepo, Semántica, pp. 40-45. 
Favorece el cambio semántico là relativa vaguedad de la significación de las palabras, lo cual se comprueba por las dificultades que ofrece a veces su definición. Ello va unido a la importancia de la polisemia, que también es resultado de la evolución histórica.

Al establecerse, por ejemplo, una doble evolución de algunas palabras, una por vía popular y otra por vía culta, es decir, una a través del frecuente uso del pueblo, con las consiguientes alteraciones formales, y otra mantenida en un uso culto propio de minorías y sin cambios formales tan acentuados; la primera clase de palabras pierde la relación formal que la unía con el punto de partida, conservado por vía culta. En estos casos va envuelto un proceso de especialización, por el cual alguna de las notas secundarias de la palabra originaria se desarrollan hasta hacerse decisivas en el derivado popular.

Además de la derivación ${ }^{50}$, otra motivación morfológica de las nuevas palabras es la composición ${ }^{51}$, unión de dos o mas palabras para formar otra, en la que el valor y el significado de la nueva palabra suele ser más extenso que el de la suma de sus componentes ${ }^{52}$.

Lehmann ${ }^{53}$ insiste en la importancia de los contextos en los cambios semánticos. Su reducción, ampliación o alteración, motivada por el contacto con profesionales, anuncios de periódicos, radio, televisión, y otros medios que utilizan

50 Actualmente se considera que la derivación consiste en añadir morfemas derivativos a un lexema, que puede realizar dos tipos de modificación, a saber, modificación semántica, cuando el lexema es modificado en su significado, pero no cambia de categoría gramatical (en este caso se habla de derivación homogénea); y modificación funcional, si el morfema modificante, además de producir un cambio en la significación, cambia la categoría gramatical del lexema (la derivación es ahora heterogénea); cf. F. Restrepo, Semántica, pp. 74-75; A. Martinet, «El léxico», apud Lingiüistica, pp. 229-243; y M. Alvar Ezquerra, Formación, pp: 48-64, entre otros.

5t Para la R.A.E., la composición consiste en unir dos o más palabras para formar otra, aunque, atendiéndose al método tradicional, considera las palabras en que entra preposicioncs. La razón es que para la R.A.E., los prefijos no son siempre morfemas trabados como los sufijos. Sin embargo, en las tendencias actuales, Marcos Marín, por ejemplo, no considera como forma de composición la constituida por prefijo más lexema, pues los prefijos desempeñan funciones idénticas a la de los sufijos; y en la escuela danesa se consideran dentro de los pleremas marginales. Así que debemos considerar que la composición consiste en la formación de palabras uniendo dos o más lexemas.

La composición léxica se sirve, según Alvar Ezquerra, de varios procedimientos para la creación de voces nuevas; entre ellos, la sinapsia, que da lugar a lexías complejas (estrella de mar, letra de cambio, traje de luces, etc.); la disyunción, que da origen, en este caso, a lexías compuestas en la que los dos lexemas no se han solapado gráficamente, aunque también se hayan lexicalizado (cama nido, guerra civil, cuento chino, etc.); la contraposición o unión gráfica de dos lexemas, ya sea mediante guiones (cuando hay una oposición entre ambos: germano-soviético) o sin ellos (cuando la oposición no existe: hispanoamericano); la yuxtaposición o composición de palabras en la que la fusión gráfica de los elementos participantes es total, así como su lexicalización y gramaticalización (telaraña, pasatiempo, etc.); cf. M. Alvar Ezquerra, Formación, pp. 21-48.

52 Cf. A. Fernández et alii, Semántica, pág. 56.

5.3 Cf. P. Lehmann, «El cambio en los sistemas semánticos», apud Introducción, pp. 245-268. 
palabras de acuerdo con la región donde estén, es decisiva en la producción de los cambios semánticos.

Los cambios semánticos pueden deberse también a causas históricas y culturales del entorno en el que se encuentra el sistema de comunicación lingüística.

De hecho, existe una relación entre lengua y cultura ${ }^{54}$, más que de causalidad, dinámica, en la que el individuo tiene un papel muy importante en el cambio semántico, pero pasando antes por la aprobación histórica de la sociedad, pues al adulto le interesa conservar su patrimonio lingüístico ${ }^{55}$.

$Y$ es que, de hecho, el lenguaje no sólo comunica sino que conforma nuestra imagen del mundo, configurando nuestra conducta, tanto individual como socialmente. Es el giro de lo individual a lo social que además de constituir el primer paso para el nacimiento y formulación de una nueva disciplina (la sociolingüística), sienta las bases para una nueva forma de concebir el lenguaje en la que objetivismo y humanismo se dan la mano en el «nuevo mundo técnico» que nos ha tocado vivir ${ }^{56}$.

Este proceso de interrelación tiene diversas manifestaciones lingüísticas; piénsese, como ya hemos mencionado con anterioridad, en los cambios sufridos por los objetos que, sin embargo, siguen llamándose del mismo modo, con independencia de algunas modificaciones formales si nos remontamos mucho. Como ejemplo podemos considerar el lexema carro, cuyo significado ha adquirido otros sememas para corresponder en hispanoamérica al valor de 'automóvil', de igual modo que nuestro coche ${ }^{57}$.

Igualmente, puede cambiar la manera de ser de muchas instituciones, ideas, etc., aunque sigamos empleando las mismas palabras. Se ha de tener en cuenta el cambio del mundo real y del mundo de los conceptos, que influyen en los cambios semánticos en general.

Es arriesgado prescindir de la dimensión social del lenguaje para explicar el cambio lingüístico ${ }^{58}$, puesto que, en el fondo, es la sociedad la que contribuye a

54 Aunque el lenguaje es natural, puesto que el ser humano tiene la capacidad innata para hablar, también es cultural, porque el hombre depende de sus semejantes, vive en una misma sociedad compartiendo una misma cultura. Se les puede vincular por infinitas redes de relaciones dinámicas, en las que juega un papel muy importante la noción de forma interior. Su relación se analiza también mediante la geografía linguística, que nos muestra la distribución geográfica de ciertos rasgos lingüísticos, y la geografía de la cultura, que estudia la distribución de la organización social y de ciertas manifestaciones artísticas; cf. $O$. Uribe Villegas, "Lengua, sociedad y cultura», apud Sociolingüistica, pp. 97-113.

55 Cf. O. Uribe Villegas, Sociolingüística, pág. 124.

s6. De ahí la necesidad de estudiar cómo se configura el significado en los diferentes medios de comunicación y la manera en que estos medios conforman el significado; cf. al respecto, $\mathrm{M}$. Martínez Arnaldos, Lenguaje. Las bases del pensamiento social sobre el lenguaje pueden verse en $J$. A. Villena, Fundamentos.

57 V. Lamíquiz, Lingüística, pág. 415. También puede verse Ch. Kany, Hispanoamericana.

s8 De hecho, como reconoce Labov, si esto ocurriese nos quedaríamos con un conjunto muy 
crear nuevas normas en el uso y el vocabulario de una lengua, posibilitando incluso que distintos cambios lingüísticos adquieran una relevancia especial y un uso acelerado en la sociedad. De ahí la formulación de dos tendencias basadas en distintas concepciones del lenguaje, a saber, la tendencia psicolingüistica, centrada en la comunicación de la información referencial, y la tendencia sociolingüistica. Veamos, brevemente, cada una de ellas.

Dentro de la primera, Whitney ${ }^{59}$ defiende que el habla no es un bien personal sino un bien social que pertenece no al individuo sino al miembro de la sociedad. El hombre habla no para pensar, sino para comunicar su pensamiento, movido por sus necesidades sociales.

Para H. Paul ${ }^{60}$ y H. Swett ${ }^{6 !}$, por el contrario, el lenguaje es expresión del pensamiento por medio de sonidos hablados, sin tener en cuenta el contexto social. El cambio se explicaría por rasgos individuales (pereza, negligencia) más que por rasgos sociales.

Para Meillet ${ }^{62}$, del hecho de que el lenguaje sea una institución social se sigue que la lingüística es una disciplina social $y$, por tanto, la variable para explicar el cambio lingüístico es el cambio social.

La tendencia sociolingüística sí se ocupa de los usos fácticos y afectivos del lenguaje, relacionándose más con los aspectos sociales. Un primer grupo, representado por Whitney, Schuchardt, Meillet, Vendryes, Jespersen, etc., prestan atención a los factores sociales en la explicación del cambio linguístico. Consideran relacionadas las funciones expresivas y directivas del lenguaje con la comunicación referencial, y estudian, a través de los mapas dialectales, el cambio, enfatizando la importancia de la diversidad lingüística, las lenguas en contacto, y las oleadas lingüísticas.

Un segundo grupo, formado por Trubetzkoy, Bloomfield, Hockett, Martinet, Kurylowicz, y Chomsky, entre otros, representa el ámbito asocial que centra sus estudios en los factores estructurales y psicológicos para explicar el cambio ${ }^{63}$, fruto de la interferencia disfuncional en el desarrollo normal de la lengua que se produce por el influjo de lo social ${ }^{64}$.

limitado de hechos, que nos condenaría a repetir argumentos pasados. Para la base social del cambio lingüístico puede consultarse W. Labov, Modelos, pp. 325-337. Un ejemplo interesante de la influencia de la situación sociocultural en el léxico puede verse en P. Vega, «Influencia», pp. 115-134.

59 W. D. Whitney, Language, pp. 401-404.

60 H. Paul, Principles.

51 H. Sweet, History.

62 A. Meillet, Linguistique, pp. 16-17.

63 Hoy se puede ver que el cambio en el significado de una forma es un cambio en el uso de ésta, de bido a situaciones estructurales o psicológicas que sólo se pueden conocer si la tradición histórica es favorable. El cambio semántico es, por tanto, un fenómeno complejo que, en palabras de Bloomfield, implica preferencias y abandonos y, en su punto crucial, la extensión de una forma favorecida por aplicaciones prácticas; cf. L. Bloomfield, Language, pp. 514-537.

64 Cf. W. Labov, Modelos, pág. 330. 


\subsection{Consecuencias de los cambios semánticos}

Entre las innumerables consecuencias que pueden resultar de los cambios semánticos, podemos citar las siguientes: en primer lugar, el cambio semántico puede tener por resultado un ensanchamiento o una restricción del sentido. Por el primer camino desaparecen ciertas características del objeto, esenciales al principio, y sólo algunas otras que, a veces, son secundarias, acaban predominando y proporcionando a la palabra un alcance o ampliación mucho mayor. El fenómeno contrario es la especialización. Es frecuente que muchas palabras, al ser usadas en sectores sociales especiales, a veces profesionales, adquieran un sentido más restringido a través de la evolución popular.

Por último, Ullmann ${ }^{65}$ considera los cambios en cuanto a la valoración, hablándonos de los desarrollos peyorativos de los significados.

\section{La aproximación epistemológica al cambio semántico}

La problemática planteada a lo largo de estas páginaś nos hacen recordar ${ }^{66}$ que aunque formalismo, historicismo y filología, en cierto modo, como metodologías específicas de acercamiento del discurso lingüístico hayan logrado con creces el pretendido estatuto objetivista que anhelara desde presupuestos teóricos más cercanos a las ciencias formales que a otras parcelas del saber, existe una carencia epistémica que justifica el hecho de que, frente a una lingüística que separa el signo de la cosa y lo pone en relación con otros signos en un sistema lógico cerrado (formalismo) deba unirse signo y realidad (humanismo), propiciando una apertura del sistema y, consecuentemente, la concepción de la actividad comunicativa como un auténtico sistema social.

Es el carácter ideológico del significado, que justifica el proceso de ontologización (muy cercano al relativismo, ciertamente, motivado por el descentramiento del sujeto unitario como garantía del conocimiento, lo que lleva a múltiples puntos de vista, tantos como clases) ya que se trata de comprender la realidad a partir del sistema de valores lingüísticos, de las categorías semánticas en términos de las cuales se construye en el acto comunicativo la información socialmente relevante.

La problemática resulta ser, pues, la siguiente:

— El análisis de la configuración del lenguaje sujeto en la lengua objeto a través de la evolución semántica que la sociedad ejerce sobre ésta.

- El estudio de la manera en que la sociedad a través de los medios de comunicación conforma también ese lenguaje sujeto.

65 Cf. S. Ullmann, Semántica, pp. 26I-266.

66. Cf. la reflexión que hicimos a propósito del libro del profesor Martínez Arnaldos, Lenguaje, pp. $470-474$. 
Y al igual que el problema teórico del significado ha interesado a distintas disciplinas ${ }^{67}$, la problemática planteada también ha sido abordada desde diferentes planteamientos epistemológicos, que conviene recordar llegados a este punto ${ }^{68}$.

Al ser la propia sociedad el valor determinante no sólo del proceso comunicativo en general, sino también del propio cambio semántico, cl nivel sociológico constituye el acercamiento básico e inicial. Sin embargo, puesto que hay factores psicológicos y pragmáticos que determinan el cambio semántico, debe completarse el planteamiento sociológico señalado con propuestas destinadas a precisar el comportamiento individual en la problemática señalada.

Sin embargo, y puesto que no se trata tanto de estudiar el significado en la historia sino la historia del significado a través de la evolución semántica de la lengua como un sistema de valores, el acercamiento prioritario debe ser el que desde un nivel estrictamente lingüístico aporta la lingüística del sujeto, en cuanto técnica de interpretación que nos permita comprender ideológicamente las categorías semánticas que permiten la evolución y, por consiguiente, el cambio lingüístico.

En este sentido, conviene recordar que el término ideología ha sido usado de diferentes maneras a lo largo de la historia:

- En primer lugar, como un conjunto de formas mediante las cuales los hombres toman conciencia de su realidad o experiencia social. En este sentido, la ideología como sublimación de ciertas condiciones sociales, denota una serie de idcas conscientes y se opondría a inconsciente y a la filosofía espontánca oculta en los textos ${ }^{69}$.

- Una serie de ideas confusas, opuestas al conocimiento verdadero, al saber o a la ciencia. Ahora la ideología denotaría la valoración epistemológica de un objeto y se opondría al conocimiento científico. Sería, por tanto, el conocimiento epistemológico de los datos de la experiencia (lingüística) mediante la elaboración de una serie de Propuestas Modélicas ${ }^{70}$.

Una mezcla de los dos sentidos mencionados es la que nos permite entender el cambio semántico como una respuesta a la variación ideológica de una

47 Cl. J. L. Jiménez Ruiz, «Demarcación», pp. 117-150.

(18 Seguimos, en este caso, las propuestas que pueden verse en. D. McQuail, Teoría, pp. 67-68.

(i) Entendemos por filosofía espontánea, siguiendo a Althusser, Curso, pp. 67 y ss., la modalidad que afecta a los elementos mediante los cuales el teórico de una disciplina construye su entramado particular; véase, al respecto, M. Crespillo, Historia, pp. 53 y ss.; y J. L. Jiménez Ruiz, Fundamentos, pp. 103 y ss.

71) Una vez que se ha precisado nuestro objeto de estudio (el significado de la lengua como un sistema estructurado) se trata de construir la Teoría (aquellos que se sitúen únicamente en la vertiente más inmanente y formalizada del ámbito linguístico) o el Modelo (los que estudien la vertiente objetual como paso a la caracterización sujetual) que desarrolle de manera abstracta la descripción y explicación de nuestro objeto para determinar, en el primero de los casos, la verdad, o, en el segundo, la corrección de sus planteamientos internos. 
colectividad, y las propuestas para su caracterización, la establecida en el triple plano que nos facilita:

$\left.1^{\circ}\right)$ obtener la estructura profunda del sistema semántico de la Lengua española mediante la producción glotológica no sólo de un aparato conceptual (formado por las idcas conscientes del primer sentido mencionado de la ideología), sino también nocional (constituido, en este caso, por la reflexión epistémica que constituye el segundo sentido de la ideología $)^{71}$;

$2^{\circ}$ ) explicar la estructura superficial a través de la cual se plasma el sistema semántico ante la conciencia colectiva ${ }^{72}$;

$3^{\circ}$ ) interpretar esa misma conciencia como factor determinante del cambio semántico.

En este sentido, la distinción entre estructura profunda (la que permite engendrar la estructura superficial) y estructura superficial (la que nos permite comprender la profunda, es decir, desligar to que es de lo que parece ser) posibilita la elaboración nocional del aparato glotológico que pretende explicar la estructura profunda del sistema semántico, la superficial a través de la cual se nos presenta y, finalmente, la ideología que lo suscita.

La razón es obvia ya que la ideología constituye un nivel de significación presente en cualquier tipo de mensaje, lo que posibilita no sólo formulaciones teóricas como la de Trías ${ }^{73}$, por poner un caso, sino también la lectura ideológica de los diferentes sistemas.

Tanto la Teoría como el Modelo se constituyen a partir de una serie de desarrollos específicos que vamos a llamar Propuestas Teóricas o Propuestas Modélicas, según sea la fundamentación científica o epistemológica en la que se sitúe la investigación, con los que se hace frente al acercamiento ya sea del Objeto en su totalidad o de una parte del mismo.

De hecho, puede suceder que exista una sola Propuesta Teórica o Modélica y que explique todas las facetas del Objeto, o que existan distintas Teorías y Modelos desarrollados por otras tantas Propuestas Teóricas o Modélicas. Lo cierto es que sea cual fuere el caso, podemos decir que cada uno de los conjuntos de Teorías y Propuestas Teóricas constituyen los Paradigmas de las Cicncias, frente a los conjuntos de Modelos y Propuestas Modélicas, que constituyen, en este caso, los Paradigmas de las Epistemologías.

La Ciencia del Lenguaje (en nuestro caso la Epistemología del Lenguaje) posee dos grandes paradigmas que desarrollan la vertiente objetual de la misma; a saber, el Generativo (que utiliza el Método transformacional) y el Estructural, Taxonómico o Descriptivo (que utiliza, en este caso, el Método estructural); Cf. al respecto, J. L. Jiménez Ruiz, Fundamentos, pp. 155 y ss.

7 Seguimos aquí la distinción ya clásica entre nociones, en cuanto unidad específica del discurso epistemológico y conceptos, en cuento unidad específica del discurso científico.

72 De hecho, la ideología enlaza con las condiciones y la forma de la comunicación social y como expresa manifiestamente Voloshinov, Signo ideológico, pág. 24:

«...la existencia del signo no es otra cosa que la materialización de esa comunicación [social], y de esa naturaleza son todos los signos ideológicos».

73 Cf. E. Trías, Teoria. 
En este proceso de lectura ideológica del cambio semántico deben tenerse en cuenta las siguientes reflexiones ${ }^{74}$ :

$1^{\circ}$ ) La información ideológica no es un acto de comunicación sino de metacomunicación, es decir, operante en el nivel connotativo y no en el denotativo. $2^{\circ}$ ) La ideología supone un sistema de reglas semánticas que expresan un determinado nivel de organización no sólo de los significados sino también de los mensajes y desde el que es posible generar nuevos mensajes.

$3^{\circ}$ ) La clave para comprender cómo los mensajes controlan la conducta no está en el contenido explícito del mensaje, sino en la organización de los mismos.

$4^{\circ}$ ) Por tanto, el análisis ideológico debe centrarse en la búsqueda de las bases semánticas en términos de las cuales se construye en el acto comunicativo la información socialmente relevante.

Así, no sólo reconstruiremos la sociedad, el mundo surgido a través de la interacción ${ }^{75}$, sino que entenderemos con mayor claridad el proceso que determina los cambios semánticos $\mathrm{y}$, por consiguiente, la historia de nuestra lengua ${ }^{76}$.

\section{Referencias bibliográficas}

ALTHUSSER, L., Curso de filosofía para cientificos, Laia, Barcelona, 1975. ALVAR, M. (coord.), El lenguaje político, Instituto de Cooperación Iberoamericana, Madrid, 1987.

ALVAR EZQUERRA, M., Lexicología y lexicografía, Almar, Salamanca, 1983. ALVAR EZQUERRA, M. et alii, Industrias de la Lengua, Fundación Germán Sánchez Ruipérez y ed. Pirámide, Madrid, 1991.

ALVAR EZQUERRA, M., La formación de palabras en español, Arco Libros, Madrid, 1993.

ALVAR EZQUERRA, M. \& VILLENA PONSODA, J. A. (eds.), Estudios para un corpus del español, Universidad de Málaga, Málaga, 1994.

ANDERSON, J. M., Aspectos estructurales del cambio lingüístico, Gredos, Madrid, 1977.

BLOOMFIELD, L., Language, Allen y Unwin, Londres, 1976.

CAMPO, S. del (ed.), Tratado de Sociología, I y II, Taurus, Madrid, 1984.

CASARES, J., Introducción a la lexicografía moderna, CSIC, Madrid, 1969.

CASAS, M., «Hacia una caracterización semántica de la terminología lingüística», Estudios de Lingüística, 10 (1994-1995), pp. 45-65.

COSERIU, E., Principios de semántica estructural, Gredos, Madrid, 1977.

74 Véase, para todo ello, las reflexiones de E. Verón et alii, Lenguaje, pp. 140-143.

75 Cf. M. J. Úriz Pemán, «Factores sociales», pág. 57 y ss.

$211-225$

Sus presupuestos generales pueden verse en nuestro trabajo «Historia de la lengua», pp. 
COSERIU, E., Lecciones de lingüistica general, Gredos, Madrid, 1981.

CRESPILLO, M., Historia y mito de la Lingüística transformatoria, Taurus, Madrid, 1986.

CRESPILLO, M., «La miseria de la Filología», Analecta Malacitana, XVII, 2 (1994), pp. 271-288.

ESPEJO MURIEL, M. del M., Los nombres de los colores en español, Universidad de Granada, Granada, 1990.

FERNÁNDEZ GONZÁLEZ, A. et alii, Introducción a la semántica, Cátedra, Madrid, 1977.

FERNÁNDEZ SEVILLA, J., «Sinonimia y polisemia: implicaciones didácticas», Actas del IV Simposio de Lengua y Literatura españolas para profesores de Bachillerato, Universidad de Granada, Granada, 1983, pp. 41-55.

GARCÍA ASENSIO, M ${ }^{a}$ A. \& YUFERA, I., «Sobre la evolución semántica de algunas palabras del español que designan unidades de comunicación verbal», Revista Española de Lingüistica, 23, 2 (1993), pp. 359-373.

GARRIDO MEDINA, J. C., «El cambio semántico en el léxico político: el verbo detentar», apud ALVAR, M. (coord.), Lenguaje, pp. 136-146.

GEERAERTS, D., «Reclassifying semantic change», Quaderni di Semantica, 4 (1983), pp. 217-240.

GIMENO, F., Dialectología y sociolingüística españolas, Universidad de Alicante, Alicante, 1993.

GIMENO, F., Sociolingüística histórica (siglos X-XII), Visor y Universidad de Alicante, Madrid, 1995.

JIMÉNEZ RUIZ, J. L., «Propuestas de demarcación disciplinaria en el análisis dialéctico del significado», Estudios de Lingüística, 9 (1993), pp. 117-150.

JIMÉNEZ RUIZ, J. L., «Sobre Martínez Arnaldos, M., Lenguaje, texto y massmedia, Universidad de Murcia, Murcia, 1990», Verba, 20 (1993), pp. 470-474.

JIMÉNEZ RUIZ, J. L., «Fundamentos epistémicos para una concepción sociolingüística de la historia de la lengua», Lexis, XVIII, 2 (1994), pp. 211 225.

JIMÉNEZ RUIZ, J. L., «El proyecto lexicográfico de M. Alvar Ezquerra: entre la modernidad y el didactismo», Epos, X (1994), pp. 493-502.

JIMÉNEZ RUIZ, J. L., Semántica sintomática. Propuestas para una hermenéutica comprensiva del sentido, Universidad de Alicante, Alicante, 1994.

JIMÉNEZ RUIZ, J. L., Fundamentos epistémicos de Lingüística española, Gamma, Alicante, 1995.

JIMÉNEZ RUIZ, J. L., «El significado en la historia o la historia del significado», apud LLOPIS GANGA J. \& TORNEL SALA, J. L., Dinamismo, pp. 9-17.

JIMÉNEZ RUIZ, J. L., «El carácter semántico del orden decimonónico y sus repercusiones epistémicas», apud GIMÉNEZ, E. \& LOZANO, M . A . \& RIOS, J. (eds.), Españoles en Italia e italianos en España, Universidad de Alicante, Alicante, 1996, pp. 89-97. 
JIMÉNEZ RUIZ, J. L., «Propuesta de caracterización semántica de la lengua española: entre la teoría y la empiricidad», Analecta Malacitana, XIX, 2, (1996), pp. 441-454.

KANY, Ch. E., Semántica hispanoamericana, Aguilar, Madrid, 1962.

LABOV, W., «Come usare il presente per spiegare il passato», apud Il continuo e il discreto nel linguaggio, Il Mulino, Bolonia, 1977, pp. 121-158.

LABOV, W., Modelos sociolingüisticos, Cátedra, Madrid, 1983.

LAMÍQUIZ, V., Lingüistica española, PUS, Sevilla, 1975.

LEHMANN, W. P., «El cambio en los sistemas semánticos», apud Introducción a la Lingüistica histórica, Gredos, Madrid, 1969, pp. 245-268.

LYONS, J., Semántica, Teide, Barcelona, 1980.

LLOPIS GANGA, J. \& TORNEL SALA, J. L., Dinamismo lingüístico: la evolución sociolingüística de la episteme medieval, Editorial Club Universitario, Alicante, 1996.

MAINGUENEAU, D., Introducción a los métodos de análisis del discurso, Hachette, Buenos Aires, 1980.

MARTINET, A., La lingüística, Anagrama, Barcelona, 1972.

MARTÍNEZ ARNALDOS, M., Lenguaje, texto y mass-media, Universidad de Murcia, Murcia, 1990.

MATORÉ, G., La Méthode en lexicologie. Domaine français, Didier, París, 1973.

MAURO, T. de, Introduzione a la semantica, Laterza, Bari, 1965.

MAURO, T. de, Minisemántica, Gredos, Madrid, 1986.

McQUAIL, D., Introducción a la teoría de la comunicación, Paidós, Buenos Aires, 1983.

MEILLET, A., Linguistique Historique et Linguistique Générale, Société Linguistique de Paris, París, 1921.

MONDÉJAR, J., «Sobre palabras y términos», apud ESPEJO MURIEL, Mª del M., Colores, pp. 11-15.

MÜLLER, Ch., Estadística lingüística, Gredos, Madrid, 1973.

PAUL, H., Principles of the History of Language, MacMillan, Nueva York, 1989.

PORZIG, W., «Cambios lingüísticos», apud El mundo maravilloso del lenguaje, Gredos, Madrid, 1974, pp. 294-357.

RAE, Diccionario de la lengua española, Madrid, 1992.

RESTREPO, F., Diseño de semántica general, Norma, Colombia, 1938.

RODRÍGUEZ ZÚÑIGA, L., «El desarrolllo de la teoría sociológica», apud CAMPO, S. del (ed.), Sociología, pp. 15-56.

ROSSI, T. M., «Denominar lo nuevo y volver a denominarlo: un problema a raíz del descubrimiento», Lingüística Española Actual, 14, 2 (1992), pp. 243-255.

SALVADOR, G., Semántica y lexicología del español, Paraninfo, Madrid, 1985. SAUSSURE, F. de, Curso de Lingüística General, Losada, Buenos Aires, 1979. 
SNELL, B., «Cambios de significación», apud Estructura del lenguaje, Gredos, Madrid, 1966, pp. 164-168.

SWEET, H., The History of Language, L. M. Dent, Londres, 1900.

TESO MARTÍN, E. del, «Cambio semántico, impropiedad y eufemismo», Verba, 15 (1988), pp. 183-204.

TRÍAS, E., Teoría de las ideologías, Península, Barcelona, 1970.

URIBE VILLEGAS, O., «Lengua, sociedad y cultura», apud Sociolinguiística: una introducción a su estudio, Universidad Nacional Autónoma de México, México, 1970, pp. 97-113.

ÚRIZ PEMÁN, M. J., «Reflexiones críticas sobre la influencia de los factores sociales en el individuo", Letras de Deusto, 57 (1993), pp. 55-75.

VEGA, P., «Notas sobre la influencia de la situación sociocultural en el léxico: la palabra feminismo (1940-1945)», Anuario de Lingüística Hispánica, 6 (1990), pp. 115-134.

VERÓN, E. et alii, Lenguaje y conunicación de masas, Nueva Visión, Buenos Aires, 1976.

VOLOSHINOV, L. V., El signo ideológico y la filosofía del lenguaje, Nueva Visión, Buenos Aires, 1976.

TRUJILLO, R., «Gramática, lexicología y semántica», Revista Española de Lingüistica, II, 1 (1972), pp. 103-109.

TRUJILLO, R., El campo semántico de la valoración intelectual en español, Universidad de La Laguna, La Laguna, 1970.

ULLMANN, S., Lenguaje y estilo, Aguilar, Madrid, 1968.

ULLMANN, S., Semántica: introducción a la ciencia del significado, Aguilar, Madrid, 1967.

VILlENA, J. A, Fundamentos del pensamiento social sobre el lenguaje, Ágora, Málaga, 1992.

WHITNEY, W. D., Language and the Study of Languages, Scribiner's, Nueva York, 1901.

WOTJAK, G., Investigaciones sobre la estructura del significado, Gredos, Madrid, 1979.

ZGUSTA, L., Manual of Lexicography, Academia-Mouton, Praga-La HayaParís, 1971. 